\title{
Thermal roughening of $\{001\}$ surfaces
}

\author{
Harold J. W. Zandvliet,* Frank R. van Dijk, and Bene Poelsema \\ Solid State Physics group and MESA+Research Institute for Nanotechnology, University of Twente, P.O. Box 217, \\ 7500 AE Enschede, The Netherlands
}

(Received 24 May 2005; revised manuscript received 1 August 2005; published 21 September 2005)

\begin{abstract}
Within the framework of a solid-on-solid model that incorporates nearest- $(\varepsilon)$ and next-nearest-neighbor $(\delta)$ interactions we have determined the free energy of the high-symmetry steps on a (001) surface of a cubic crystal. We have found a simple expression that allows one to determine the thermal roughening temperature $T_{R}$ of a $(001)$ surface $\left(2 e^{-(\varepsilon / 2+\delta) / k_{b} T_{R}}-e^{-(\varepsilon+2 \delta) / k_{b} T_{R}}+2 e^{-(\varepsilon+\delta) / k_{b} T_{R}}=1\right)$. In a more refined analysis we have explicitly included step-edge overhangs. This results in a slightly lower thermal roughening temperature. Our results are also applicable to the two-dimensional Ising spin system.
\end{abstract}

DOI: 10.1103/PhysRevB.72.113412

PACS number(s): 68.35.Md

\section{INTRODUCTION}

Surface steps are of utmost importance for the understanding of the thermodynamic behavior of surface morphology. Previous studies have shown that surface steps play a key role in many equilibrium processes on surfaces, such as thermal roughening, faceting, evolution of the equilibrium shape of islands and vacancy islands as a function of temperature, temporal and spatial step-edge fluctuations, and Ostwald ripening. Despite this importance a detailed knowledge of the exact value of the step edge free energy is, however, often still lacking. The most straightforward way to extract information about the step free energy is by a statistical analysis of real-space images of the step-edge meandering taken with, for instance, a scanning tunneling microscope or a low-energy electron microscope. ${ }^{1-6}$ Usually a simple solidon-solid model that incorporates only nearest-neighbor interactions, in which step-edge overhangs are ignored, is sufficient to obtain an accurate temperature dependence of the step free energy at low temperatures. The reason for this proper description is that step-edge meandering, leading to a reduction of the step free energy with increasing temperature, involves only small step-edge excursions-i.e., mainly thermal generation of single kinks - at low temperatures. At higher temperatures also larger step excursions, such as multiple kinks, need to be taken into account as well. ${ }^{2,7-10}$ In principle the cubic (001) Kossel crystal with nearestneighbor interaction can be mapped onto the twodimensional (2D) spin system. The nearest-neighbor interaction between the atoms can be associated with the Ising parameter. In 1944 Onager $^{11}$ derived an expression for the boundary tension between two regions of opposite spins. As pointed out by Rottman and Wortis ${ }^{8}$ this expression is also valid for the free energy of steps of a simple cubic Kossel crystal.

It is the aim of this work to determine the thermal roughening temperature of a simple cubic surface having nearestand next-nearest-neighbor interactions. Next we extend our model and incorporate overhangs in the step edges as well. The latter results in a slight reduction of the thermal roughening temperature. Moreover, our results are also valid for the boundary tension between two regions of opposite spins in the 2D Ising system. ${ }^{9,11}$

\section{MODEL}

We consider a simple square lattice with nearest-neighbor distance $a$. We introduce an attractive nearest-neighbor interaction $(\varepsilon)$ and a next-nearest-neighbor interaction $(\delta)$ that can either be attractive, zero, or repulsive. For some (001) surfaces, such as $\mathrm{Cu}$ (Refs. 12-15) and Si (Ref. 2), the nextnearest-neighbor interaction has a nonzero value, whereas for other materials the next-nearest-neighbor interaction can be ignored [e.g., $\mathrm{Ge}(001)]$. $^{16,17}$

The $[110]$ and $[-110]$ directions run along the closepacked directions of the (001) surface. The step-edge energy of these close-packed steps (per unit step-edge length $a$ ) can be found by counting the number of broken bonds per stepedge atom. Per step-edge atom one nearest-neighbor bond and two next-nearest-neighbor bonds need to be broken in order to create two step edges of unit length $a$. The step-edge formation energy is thus half of the sum of these interaction energies-i.e., ${ }^{4}$

$$
E_{[110]}=\frac{\varepsilon}{2}+\delta
$$

In an analogous way the kink formation energy for a kink in a [110] step with a length of $n$ units can easily be found,

$$
E_{k i n k}(n)=n \frac{\varepsilon}{2}+(n-1) \delta .
$$

The partition function of an elementary step segment of a [110]-oriented step is found by summing over all possible configurations, ${ }^{2}$

$$
Z_{[110]}=e^{-(\varepsilon+2 \delta) / 2 k_{b} T}\left(1+2 \sum_{n=1}^{\infty} e^{-[n \varepsilon+2(n-1) \delta] / 2 k_{b} T}\right) .
$$

The factor of 2 in Eq. (3) arises because kinks can point inward or outward. The step free energy is given by

$$
\begin{aligned}
F_{[110]} & =-k_{b} T \ln \left(Z_{[110]}\right) \\
& =\frac{\varepsilon}{2}+\delta-k_{b} T \ln \left[1+\frac{2 e^{-\varepsilon / 2 k_{b} T}}{1-e^{-(\varepsilon / 2+\delta) / k_{b} T}}\right] .
\end{aligned}
$$

With increasing temperature the step free energy will even- 
tually vanish, leading to a spontaneous generation of the steps on the surface. This roughening temperature $T_{R}$ can be extracted from the equation

$$
2 e^{-(\varepsilon / 2+\delta) / k_{b} T_{R}}-e^{-(\varepsilon+2 \delta) / k_{b} T_{R}}+2 e^{-(\varepsilon+\delta) / k_{b} T_{R}}=1 .
$$

The above expression can also be used to determine the order-disorder phase transition temperature of the 2D Ising system with nearest- and next-nearest-neighbor interactions. For a vanishing next-nearest-neighbor interaction the original result of the order-disorder phase transition temperature by Onsager ${ }^{11}$ for the $2 \mathrm{D}$ Ising system is, of course, recovered $\left[\sinh \left(\varepsilon / 2 k T_{R}\right)=1\right]$.

Next we slightly refine our approach by including overhangs in the steps as well. Including overhangs will lead to additional terms in the partition function $Z_{[110]}$ [see Fig. 1(a)]. If we include short overhangs [up to an additional step-edge length of $5 a$ per unit step edge measured in the [110] direction-i.e., $a$; see Fig. 1(a)] we find

$$
\begin{aligned}
Z_{[110]}^{*}= & Z_{[110]}+2\left(e^{-(5 \varepsilon+6 \delta) / 2 k_{b} T}+e^{-(6 \varepsilon+6 \delta) / 2 k_{b} T}\right. \\
& \left.+2 e^{-(6 \varepsilon+8 \delta) / 2 k_{b} T}\right) .
\end{aligned}
$$

It should be pointed out here that we have only considered the eight shortest overhang configurations. This is a very reasonable simplification because the probability of finding an overhang configuration depends heavily on its length [see Eq. (4)]. The step-edge free energy of the [110] step is given by

$$
F_{[110]}=-k_{b} T \ln \left(Z_{[110]}^{*}\right) .
$$

In order to derive an expression for a [010]-oriented step we apply a similar procedure. If step-edge overhangs are excluded, the partition function of a [010]-oriented step is given by ${ }^{12}$ [see Fig. 1(b)]

$$
Z_{[010]}=e^{-(\varepsilon+\delta) / 2 k_{b} T}+e^{-(\varepsilon+2 \delta) / 2 k_{b} T} .
$$

Here we have only considered the two energetically most favorable pathways, because all the other pathways involve significantly more step-edge length and are therefore much higher in energy [see Fig. 1(b)]. This approximation is a good one at low temperatures; however, with increasing temperature the free energy to generate additional step lengths decreases and the probability that step-edge overhangs occur increases. Therefore, it is appropriate to include overhangs as well. In the corresponding partition function $Z_{[010]}^{*}$, we have included short overhangs (again the eight shortest overhang configurations up to an additional increase in step-edge length of $4 a$ units per unit step-edge length measured in the [010] direction-i.e., $\left.\frac{1}{2} a \sqrt{ } 2\right)$ :

$$
\begin{gathered}
Z_{\text {overhangs }}=e^{-(3 \varepsilon+3 \delta) / 2 k_{b} T}+e^{-(3 \varepsilon+4 \delta) / 2 k_{b} T}+e^{-(5 \varepsilon+8 \delta) / 2 k_{b} T} \\
+2 e^{-(5 \varepsilon+6 \delta) / 2 k_{b} T}+e^{-(5 \varepsilon+5 \delta) / 2 k_{b} T}+2 e^{-(5 \varepsilon+7 \delta) / 2 k_{b} T}, \\
Z_{[010]}^{*}=Z_{[010]}+Z_{\text {overhangs. }}
\end{gathered}
$$

The step-edge free energy of the [010] step is given by
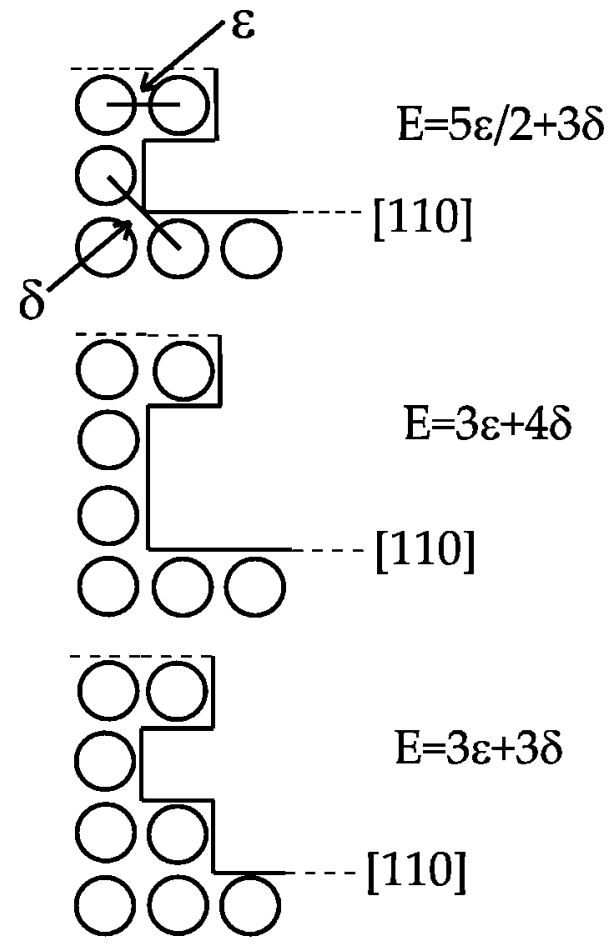

(a)
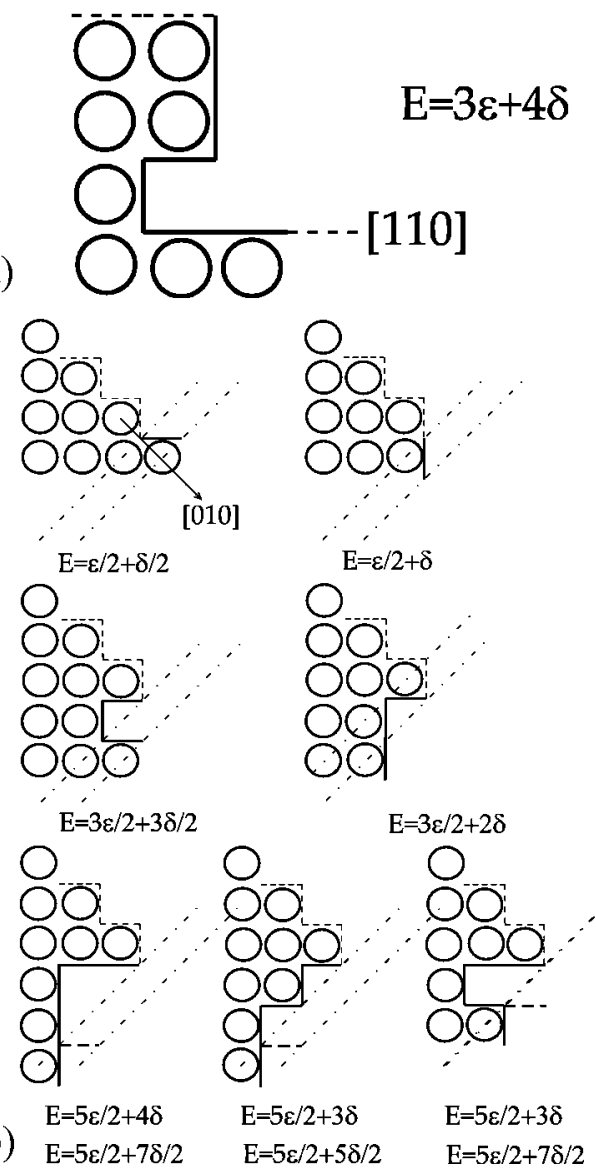

FIG. 1. (a) Schematic diagram of a step-edge overhang of a [110] step. The formation energy for each overhang configuration is given. (b) Schematic diagram of a step-edge overhang of a [010] step. The formation energy for each overhang configuration is given. 


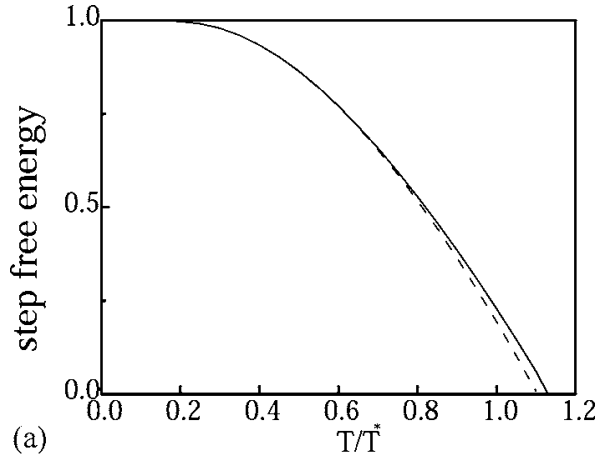

(a)

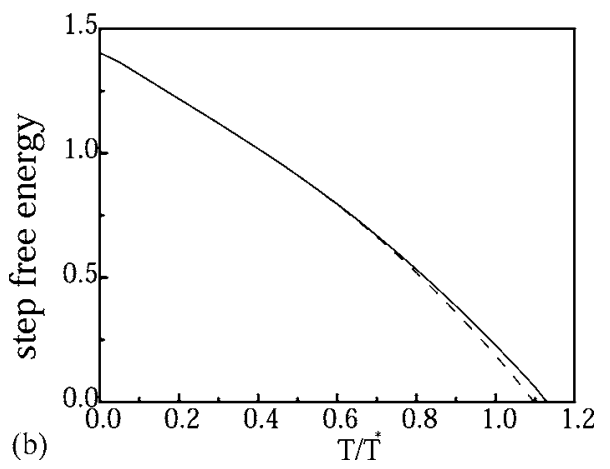

FIG. 2. (a) Plot of the (scaled) [110] step(-edge) free energies versus (scaled) temperature. The solid line refers to the well-known Onsager expression of the edge free energy (boundary tension) of the $2 \mathrm{D}$ Ising system. The dotted line refers to the step free energy in the case step-edge overhangs are included (the next-nearestneighbor interaction is set to zero). The difference in the two curves can be fully ascribed to the incorporation of overhangs. The step (-edge) free energies and temperature are scaled with $\varepsilon=2 k_{b} T^{\star}$ and $T^{\star}$, respectively. (b) Plot of the (scaled) [010] step(-edge) free energies versus (scaled) temperature. The solid line refers to the wellknown Onsager expression of the edge free energy (boundary tension) of the 2D Ising system. The dotted line refers to the step free energy in the case step-edge overhangs are included (the nextnearest-neighbor interaction is set to zero). The difference in the two curves can be fully ascribed to the incorporation of overhangs. The step(-edge) free energies and temperature are scaled with $\varepsilon$ $=2 k_{b} T^{\star}$ and $T^{\star}$, respectively.

$$
F_{[010]}=-k_{b} T \ln \left(Z_{[010]}^{*}\right) .
$$

\section{RESULTS AND DISCUSSION}

In Figs. 2 and 3 plots of the step free energies versus temperature are shown. The step free energies and temperature are scaled with $\varepsilon=2 k_{b} T^{\star}$ and $T^{\star}$, respectively. First, we restrict ourselves to nearest-neighbor interactions and compare our results with the 2D Ising model. In Figs. 2(a) and 2(b) the well-known expressions for the boundary tension between two regions of opposite spin (hereafter denoted as Onsager expressions) (Refs. 8, 9, and 11) and our results are plotted. The solid lines refer to the well-known Onsager expressions of the edge free energy (boundary tension) of the 2D Ising system along the [110] and [010] directions. ${ }^{8,11}$ The dotted lines refer to our result [Eqs. (7) and (10) with $\delta=0]$.

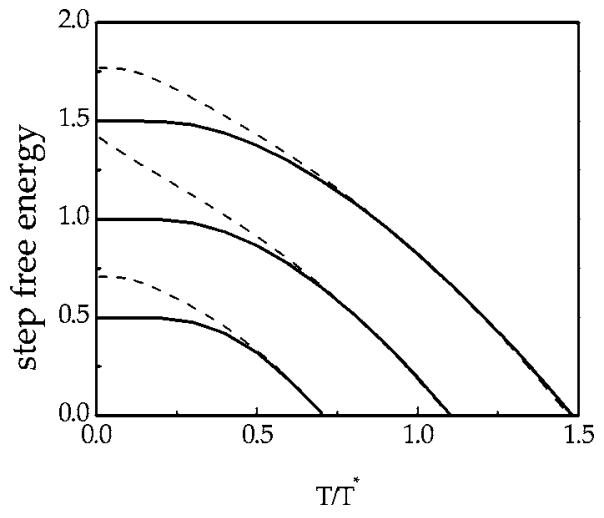

FIG. 3. Plot of the (scaled) [110] and [010] step free energies versus (scaled) temperature. Overhangs in both step edges are included. The step free energies and temperature are scaled with $\varepsilon$ $=2 k_{b} T^{\star}$ and $T^{\star}$, respectively. Solid lines refer to the [110] steps, and the dotted lines refer to the [010] steps. Upper set of curves $(\delta$ $=\varepsilon / 4$-i.e., an attractive next-nearest-neighbor interaction), middle set of curves $(\delta=0)$, and lower set of curves $(\delta=-\varepsilon / 4$-i.e., a repulsive next-nearest-neighbor interaction).

If we ignore overhangs in the [110]-oriented step, our result [using the partition function as given in Eq. (3)] collapses onto the $2 \mathrm{D}$ Ising result. Hence the difference in both curves can be fully explained by the incorporation of overhangs in the step. The effect at low or even moderate temperatures is, however, relatively small. With increasing temperature the effect becomes more pronounced. Eventually the (001) surface will roughen (vanishing of the step-edge free energy). Incorporation of overhang in the step reduces the thermal roughening temperature of the (001) surface by about $3 \%$. Next we introduce a nonzero next-nearest-neighbor interaction. This next-nearest-neighbor interaction might be attractive or repulsive, in contrast to the nearest-neighbor interaction, which should always be attractive. In Fig. 3 we present the main result of our work. The solid lines refer to the [110] steps, whereas the dotted lines refer to the [010] steps [upper set of curves (attractive next-nearest-neighbor interaction, $\delta$ $=\varepsilon / 4)$, middle set of curves $(\delta=0)$, and lower set of curves (repulsive next-nearest-neighbor interaction, $\delta=-\varepsilon / 4)]$. The roughening temperature increases (decreases) significantly due to the incorporation of an attractive (repulsive) nextnearest-neighbor interaction. This makes prefect sense because the [110] and [010] step free energies depend on the strength of the next-nearest-neighbor interaction. We see an almost perfect collapse of the [110] and [010] step free energy curves for elevated temperatures. The latter implies that the (001) surface roughens in an isotropic manner and that the 2D equilibrium shape of an island (or vacancy island) will exhibit a perfect circular shape at elevated temperatures. At zero temperature the $2 \mathrm{D}$ equilibrium is a perfect square for a repulsive or vanishing next-nearest-neighbor interaction and an octahedron for an attractive next-nearest-neighbor interaction.

\section{CONCLUSIONS}

In summary, we have derived an expression for the thermal roughening temperature of a square lattice with nearest- 
and next-nearest-neighbor interactions. For a vanishing nextnearest-neighbor interaction the original result of the orderdisorder phase transition temperature of the 2D Ising spin system by Onsager is recovered. Subsequently, we have explicitly included overhangs in the steps leading to a slight reduction of the thermal roughening temperature. These findings can also be applied to the 2D Ising system. We have found a boundary tension between two regions of opposite spins that is more accurate than the boundary tension found by Onsager.

\section{ACKNOWLEDGMENT}

This work is financially supported by the Stichting voor Fundamenteel Onderzoek der Materie (FOM).
*Electronic address: h.j.w.zandvliet@utwente.nl

${ }^{1}$ H.-C. Jeong and E. D. Williams, Surf. Sci. Rep. 34, 171 (1999).

${ }^{2}$ H. J. W. Zandvliet, Rev. Mod. Phys. 72, 593 (2000).

${ }^{3}$ B. S. Swartzentruber, Y.-W. Mo, R. Kariotis, M. G. Lagally, and M. B. Webb, Phys. Rev. Lett. 65, 1913 (1990).

${ }^{4}$ H. J. W. Zandvliet, H. B. Elswijk, E. J. van Loenen, and D. Dijkkamp, Phys. Rev. B 45, 5965 (1992).

${ }^{5}$ N. C. Bartelt, T. L. Einstein, and E. D. Williams, Surf. Sci. 240, L591 (1990); see also N. C. Bartelt, J. L. Goldberg, T. L. Einstein, and E. D. Williams, ibid. 273, 252 (1992); N. C. Bartelt, T. L. Einstein, and E. D. Williams, ibid. 276, 308 (1992).

${ }^{6}$ N. C. Bartelt and R. M. Tromp, Phys. Rev. B 54, 11731 (1996).

${ }^{7}$ H. J. W. Zandvliet and B. Poelsema, Phys. Lett. A 333, 142 (2004).

${ }^{8}$ C. Rottman and M. Wortis, Phys. Rev. B 24, 6274 (1981).
${ }^{9}$ R. K. P. Zia and J. E. Avron, Phys. Rev. B 25, 2042 (1982); M. E. Fisher and A. E. Ferdinand, Phys. Rev. Lett. 19, 169 (1967).

${ }^{10}$ T. J. Stasevich, T. L. Einstein, R. K. P. Zia, M. Giesen, H. Ibach, and F. Szalma, Phys. Rev. B 70, 245404 (2004).

${ }^{11}$ L. Onsager, Phys. Rev. 65, 117 (1944).

${ }^{12}$ R. Van Moere, H. J. W. Zandvliet, and B. Poelsema, Phys. Rev. B 67, 193407 (2003).

${ }^{13}$ H. J. W. Zandvliet, R. Van Moere, and B. Poelsema, Phys. Rev. B 68, 073404 (2003).

${ }^{14}$ M. Giesen, C. Steimer, and H. Ibach, Surf. Sci. 471, 80 (2001).

${ }^{15}$ S. Dieluwiet, H. Ibach, M. Giesen, and T. L. Einstein, Phys. Rev. B 67, 121410(R) (2003).

${ }^{16}$ H. J. W. Zandvliet, Phys. Rev. B 61, 9972 (2000).

${ }^{17}$ H. J. W. Zandvliet, Phys. Rep. 388, 1 (2003). 